\section{Efecto de la rinitis y el asma en el ausentismo y rendimiento laboral y escolar en una población del trópico latinoamericano}

Jorge Sánchez ${ }^{1}$, Javier Estarita², Carolina Salemi ${ }^{3}$

\section{Resumen}

ANTECEDENTES: el asma y la rinitis son las enfermedades respiratorias crónicas más frecuentes. Su alto efecto clínico se asocia con pérdidas de días laborales y menor rendimiento.

OBJETIVO: evaluar el efecto de la rinitis y el asma como causa de ausentismo y bajo rendimiento laboral en una población de adultos y niños.

MATERIAL Y MÉTODO: estudio trasversal, realizado en 10 escuelas de dos ciudades de Colombia. Se invitó a participar a población estudiantil entre 6 y 17 años y a los empleados mayores de 18 años de edad. A todos los participantes se les realizó un cuestionario que evaluó parámetros relacionados con la historia clínica y se hizo una revisión de las calificaciones (matemáticas y español), hojas de vida y número de ausentismos durante el último año académico completado.

RESULTADOS: aceptaron participar 1,413 personas. La frecuencia de rinitis, asma o ambas fue de $36 \%$. Hubo mayor frecuencia de ausentismo escolar en la población infantil con síntomas respiratorios (2.8 vs 1.2 días/año/paciente, p menor a 0.01) y hubo menor rendimiento académico (reprobó: 17 vs 9\%, p menor a 0.05) y laboral (0.43 vs 0.27 p menor a 0.05) en la población sintomática. Observamos que los pacientes con buen apego al tratamiento farmacológico tenían menor ausentismo y mejor rendimiento.

CONCLUSIÓN: la rinitis y el asma se asocian con menor desempeño en la población infantil y adulta, al igual que con mayor ausentismo en la escuela; sin embargo, el tratamiento adecuado puede mejorar el control clínico y reducir el efecto negativo a nivel laboral.

PALABRAS CLAVE: alergia, aprendizaje, ausentismo, asma, rendimiento escolar, rendimiento psicomotor, rinitis.
${ }^{1}$ Grupo de Alergología Clínica y Experimental (GACE), IPS Universitaria, Universidad de Antioquia, Medellín Colombia.

${ }^{2}$ Fundación Hospital San Carlos, Bogotá, Colombia.

${ }^{3}$ Javesalud IPS, Bogotá, Colombia.

Recibido: 29 de agosto 2015

Aceptado: 14 de diciembre 2015

Correspondencia

Dr. Jorge Sánchez

jotamsc@yahoo.com

Este artículo debe citarse como Sánchez J, Estarita J, Salemi C. Efecto de la rinitis y el asma en el ausentismo y rendimiento laboral y escolar en una población del trópico latinoamericano. Rev Alerg Méx 2016 ene-mar;63(1):32-40. 


\section{Rhinitis and asthma as a cause of absenteeism and poor work/school performance in a population from Latin- American tropic.}

Jorge Sánchez ${ }^{1}$, Javier Estarita², Carolina Salemi ${ }^{3}$

\begin{abstract}
BACKGROUND: Asthma and rhinitis are the most frequent chronic respiratory diseases. Their high impact is associated with the loss of working days, as well as a decrease in academic performance.

OBJECTIVE: To assess and compare the impact of rhinitis and asthma as causes of absenteeism and low work performance in a population of children and adults.

MATERIAL AND METHOD: A cross sectional study was performed in 10 schools of two cities in Colombia. The student population between 6 to 17 years, as well as the faculty staff over 18 were invited to participate. All of the participants filled a questionnaire assessing parameters related to clinical background, and a review of scores in Math and Spanish, curriculum vitae and number of missed day during the last academic period completed was performed.
\end{abstract}

RESULTS: A total of 1,413 participants were enrolled. The frequency of asthma and/or rhinitis was 36\%, there was a greater frequency of absenteeism in the population of children with respiratory symptoms (2.8 vs 1.2 days/year/patient, $\mathrm{p}<0.01)$ and there was both a lower academic (failure in $17 \%$ vs $9 \%, \mathrm{p}<0.05)$ and work performance $(0.43$ vs $0.27, \mathrm{p}<0.05)$ in the symptomatic population. We observed that those patients with good adherence to pharmacologic treatment displayed lower absenteeism and an improved performance.

CONCLUSION: Both rhinitis and asthma are associated with lower performance in children and adults and absenteeism in the school; however, adequate treatment may improve clinical control and reduce backlash against job performance.

KEYWORDS: allergy; learning; absenteeism; asthma; school performance; psychomotor performance; rhinitis
${ }^{1}$ Grupo de Alergología Clínica y Experimental (GACE), IPS Universitaria, Universidad de Antioquia, Medellín, Colombia.

2 Fundación Hospital San Carlos, Bogotá, Colombia.

${ }^{3}$ Javesalud IPS, Bogotá, Colombia.

\section{Correspondence}

Dr. Jorge Sánchez

jotamsc@yahoo.com

\section{ANTECEDENTES}

Varios estudios han demostrado la alta prevalencia de asma y rinitis en la población mundial, incluidos los países latinoamericanos. ${ }^{1-6}$ Estas enfermedades tienen un importante efecto perjudicial en la calidad de vida porque alteran el desarrollo personal y el estilo de vida de los sujetos y de sus familias. ${ }^{78}$ Existen varios estudios europeos y de Estados Unidos que muestran el 
efecto social de estas enfermedades, ${ }^{9,10}$ pero poco se ha estudiado acerca de cómo estas enfermedades pueden afectar el desempeño laboral de los pacientes y sus posibles consecuencias sociales en los países latinoamericanos, que tienen condiciones socioeconómicas diferentes. La falta de control clínico puede generar desventajas entre las personas con asma, rinitis o ambas en términos intelectuales y laborales porque estos síntomas potencialmente podrían llevar a mayor ausentismo, menor concentración $y$, por tanto, menor rendimiento en comparación con los sujetos sin estos síntomas.

El tratamiento sintomático del asma y la rinitis se centra en la administración de fármacos (antihistamínicos, esteroides inhalados o nasales, broncodilatadores) para controlar la inflamación de las vías respiratorias superiores e inferiores, ${ }^{11-13}$ pero poco se ha estudiado del efecto benéfico que este control de síntomas puede tener en los pacientes en parámetros objetivos, como la asistencia y el rendimiento laboral.

En este artículo, como desenlace primario, evaluamos si padecer rinitis, asma o ambas generaba menor rendimiento escolar o laboral y mayor ausentismo entre los pacientes mayores de seis años, en comparación con los sujetos sin estos síntomas. Como desenlace secundario, exploramos si el tratamiento farmacológico podía reducir este efecto.

\section{MATERIAL Y MÉTODO}

Estudio trasversal descriptivo. El universo total de sujetos que podían incluirse en el estudio fue de 4,123 estudiantes y 878 trabajadores (total: $n=5,001$ ); aceptaron participar 1,813 personas y 1,413 se incluyeron en el estudio (1,001 estudiantes y 412 trabajadores). Entre los 400 sujetos que aceptaron participar, pero no se incluyeron en este estudio, el principal motivo fue la falta de datos en la historia del centro, no haber estado vinculado en la institución durante el periodo de evaluación (año 2013-2014) y tener alguna comorbilidad crónica que pudiera afectar la evaluación del desenlace primario (Figura 1). Las características sociodemográficas de los pacientes excluidos eran similares a las de los pacientes que permanecieron durante el seguimiento. El universo poblacional estuvo conformado por los estudiantes mayores de seis años y los trabajadores de ocho escuelas procedentes de dos ciudades de Colombia (cuatro en Cartagena y cuatro en Bogotá). Seis de las escuelas eran públicas y dos privadas (una en cada ciudad). La recolección de los datos se realizó de enero a diciembre de 2014 por medio de evaluación médica, encuestas y datos del plantel educativo obtenidos durante el periodo escolar previo (enero a diciembre de 2013). Por medio de cuestionarios basados en el estudio ISAAC, las guías ARIA y las guías GINA (www.ginasthma. org), obtuvimos información de la existencia o no de enfermedades respiratorias, la existencia de otras enfermedades crónicas, el tratamiento farmacológico y la gravedad de los síntomas. Se recolectaron los datos de todas las personas que manifestaron estar de acuerdo en participar; sin embargo, para este artículo excluimos a todos

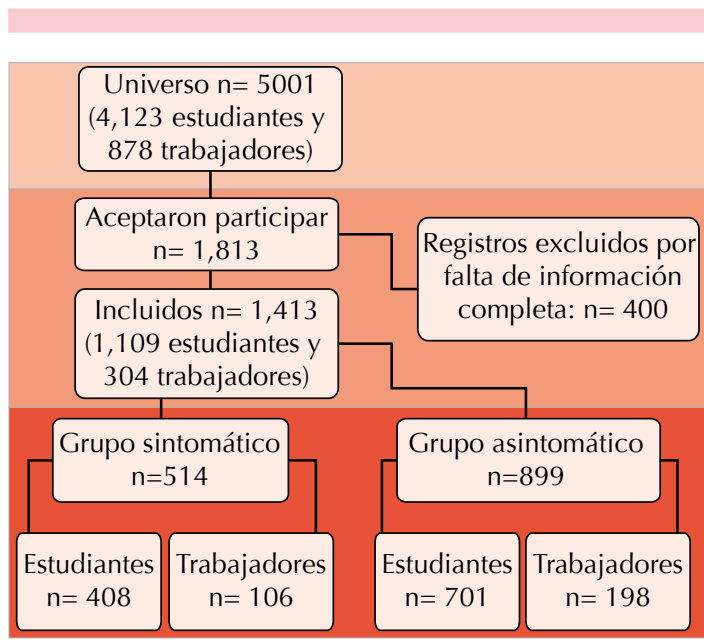

Figura 1. Flujograma del estudio. 
los sujetos con comorbilidades crónicas que pudieran influir en la interpretación de los resultados. Para esta selección utilizamos el Índice de Comorbilidad de Charlson, ${ }^{14}$ para excluir a los pacientes con comorbilidades graves que podían aumentar los costos y la gravedad de los síntomas por causas diferentes a las enfermedades estudiadas.

Para la evaluación del desenlace primario usamos como variables de medición el número de días de ausentismo, el promedio de notas de los estudiantes en matemáticas y español durante el periodo académico 2013, al igual que los comentarios, amonestaciones y otras notificaciones presentes en las hojas de vida de los trabajadores de las instituciones incluidas, que estuvieran directamente ligadas con menor desempeño laboral: no entrega de informes a tiempo, no finalización de tareas en el tiempo esperado, etc. La selección de matemáticas y español se realizó debido a que son materias con parámetros evaluativos objetivos que son estudiadas de manera trasversal durante toda la primaria y el bachillerato. Debido a las diferencias en las escalas calificativas entre las instituciones, al ser unas cualitativas y otras cuantitativas, hicimos una homogenización utilizando una escala semicuantitativa: sobresaliente $(80 \%$ o más de rendimiento esperado de acuerdo con la escala utilizada), promedio (60 a 79\%) insuficiente (reprobado, 59\% o menos). Los parámetros de evaluación usados en las escuelas fueron: examen escrito, presentaciones orales, trabajos en casa, etc. Por tanto, luego de realizar el análisis general, realizamos análisis de subgrupos para evaluar si estas diferencias eran significativas por la existencia o no de síntomas respiratorios o si, por el contrario, eran secundarias a las diferencias evaluativas e institucionales de cada escuela y de cada grado. Sólo tuvimos en cuenta los días de ausentismo relacionados con la atención en salud, licencia por enfermedad o suspensiones que indirectamente pudieran estar relacionadas con las enfermedades estudiadas.
No se incluyeron ausentismos por vacaciones, permisos para diligencias no médicas, etc.

Para evaluar el desenlace secundario realizamos un análisis estratificado entre los pacientes con asma, rinitis o ambas, de acuerdo con si el paciente recibía o no tratamiento farmacológico, que se agrupó de acuerdo con las recomendaciones de las guías GINA y ARIA.

\section{Consideraciones éticas}

Todos los pacientes - $\mathrm{O}$ en caso de ser menores de edad, sus padres o acudientes- firmaron un consentimiento informado en el que autorizaban la revisión de las datos contenidos en la institución y aceptaban completar el cuestionario. Igualmente todas las instituciones participantes dieron su aval. El Comité de Ética de la Universidad de Cartagena evaluó y otorgó el permiso para la realización de este estudio.

\section{Análisis}

Los análisis se hicieron mediante el programa SPSS versión 21 para Windows usando la prueba $U$ de Wilcoxon para análisis intergrupos, de MannWhitney para análisis intragrupos y la prueba $t$ de Student para diferencias de media en muestras independientes. Un valor de p menor a 0.05 se consideró estadísticamente significativo. Para el análisis de múltiples comparaciones utilizamos la prueba de Dunn. Las proporciones se analizaron mediante tablas de contingencias y la prueba exacta de Fisher. Debido a las diferencias en el número de sujetos por grupos, los resultados se presentan como valores absolutos o porcentajes según la variable.

\section{RESULTADOS}

\section{Descripción de los sujetos}

En esta investigación sólo incluimos como sintomáticos a los pacientes con diagnóstico médico 
de asma, rinitis o ambas. En total, 514 pacientes tenían diagnóstico de asma $(n=153)$ o rinitis $(\mathrm{n}=479), 77 \%$ de los pacientes con asma tenía rinitis, por lo que decidimos tratar la muestra como un solo grupo (grupo sintomático, Figura 1 y Cuadro 1). Observamos diferencias significativas en la distribución por sexo entre los grupos por edad y en la frecuencia de asma.

El 93\% de los pacientes con rinitis reportó que los síntomas nasales se manifestaban al menos cuatro días de la semana y $73 \%$ de los asmáticos comentó sentir síntomas bronquiales al menos dos veces por semana. Aunque 93\% de los pacientes con asma, rinitis o ambas tenía prescrito un tratamiento farmacológico, sólo 35\% lo seguía con regularidad de al menos cinco días por semana.

\section{Ausentismo}

De las exacerbaciones por cualquier motivo, la principal causa de ausentismo se asoció con enfermedades agudas o con permisos para trámites asociados con la salud en ambos grupos (89\%); en $73 \%$ de los casos, estos cuadros estaban asociados con síntomas respiratorios o eran consecuencia de ellos (por ejemplo, sinusitis).

En el grupo de sujetos sintomáticos, la frecuencia de ausentismo fue proporcionalmente similar al grupo control (días de ausencias: 716 vs 1,214, respectivamente). Sin embargo, al estratificar entre estudiantes y trabajadores, los estudiantes, especialmente entre 6 y 14 años del grupo sintomático, tenían mayor ausentismo. Debido a las diferencias en el tamaño de la muestra entre los grupos, en la Figura 2 se muestra el ausentismo de acuerdo con los días/año por número de personas en cada grupo. Entre los mayores de 18 años no hubo diferencias estadísticamente significativas $(p=0.08)$.

\section{Rendimiento laboral-escolar}

A nivel escolar se observó que el número de estudiantes con notas insuficientes (reprobado)

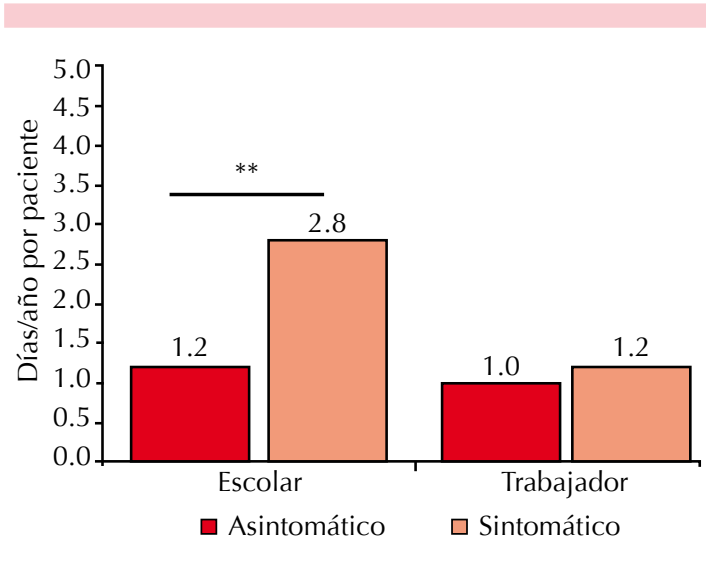

Figura 2. Ausentismo calculado de acuerdo con el número de días de ausentismo por año por número de personas en cada grupo.

${ }^{*} \mathrm{p}<0.05$.

$* * \mathrm{p}<0.01$.

Cuadro 1. Características de los pacientes

\begin{tabular}{|c|c|c|c|c|c|}
\hline & \multicolumn{4}{|c|}{ Grupo } & \multirow[b]{3}{*}{ p } \\
\hline & \multicolumn{2}{|c|}{ Estudiantes } & \multicolumn{2}{|c|}{ Trabajadores } & \\
\hline & $\begin{array}{c}\text { Sintomáticos } \\
n=408\end{array}$ & $\begin{array}{c}\text { Asintomáticos } \\
\mathbf{n}=701\end{array}$ & $\begin{array}{c}\text { Sintomáticos } \\
n=106\end{array}$ & $\begin{array}{c}\text { Asintomáticos } \\
\quad n=198\end{array}$ & \\
\hline Edad (años) & $11(6-17)$ & $12(6-17)$ & $39(18-64)$ & $40(18-63)$ & 0.03 \\
\hline Sexo femenino & $208(50 \%)$ & $364(51 \%)$ & $45(42 \%)$ & 78 (39\%) & 0.04 \\
\hline Asma & $112(27 \%)$ & 0 & $41(38 \%)$ & 0 & 0.05 \\
\hline Rinitis & 394 (96\%) & 0 & 85 (80\%) & 0 & 0.06 \\
\hline
\end{tabular}

En total, 514 pacientes (408 escolares y 106 trabajadores) tenían el diagnóstico médico de asma ( $\mathrm{n}=153)$ o rinitis $(\mathrm{n}=479) ; 98$ estudiantes y 20 adultos tenían asma y rinitis. 
en matemáticas y español era mayor en el grupo asintomático (Figura 3A). Asimismo, los trabajadores de la institución que padecían asma, rinitis o ambas tenían mayor número de amonestaciones por sujeto (Figura 3B). La principal causa de las amonestaciones fue retraso en la entrega de informes o labores (76\%).

\section{Repercusión del tratamiento farmacológico}

Entre los pacientes con síntomas respiratorios observamos que los escolares que recibían tratamiento farmacológico tenían una frecuencia significativa $(\mathrm{p}=0.04)$ de menor ausentismo (días/ año/paciente: 1.8), en comparación con quienes no lo recibían (días/año/paciente: 2.9), pero aún superior al grupo control (días/año/paciente: 1.2). Cuadro 2

Cuando analizamos si el apego al tratamiento influía en el resultado, observamos una tendencia a mejor respuesta con la administración de al menos cinco o más días por semana. De manera similar, los pacientes con tratamiento farmacológico con apego adecuado tuvieron un rendimiento superior al de los pacientes que no recibían tratamiento continuo y era similar al del grupo control. De igual manera, los pacientes trabajadores con tratamiento farmacológico adecuado tuvieron un número de amonestaciones similar al del grupo control y menor que el grupo con síntomas y sin tratamiento (Cuadro 2).

\section{DISCUSIÓN}

El asma y la rinitis son enfermedades de alta prevalencia y de carácter crónico, por lo que su efecto socioeconómico y en la salud es bastante alto para el paciente y para el sistema de salud..$^{15}$ Por esta razón, representan un problema de salud pública y es necesario evaluar el efecto que tienen para el paciente, su núcleo familiar y social.

Las variables de evaluación que utilizamos nos permiten explorar el efecto de estas enfermedades a nivel social. De manera similar a las observaciones previas realizadas en Europa y Estados Unidos, ${ }^{8,16}$ observamos que la rinitis y el

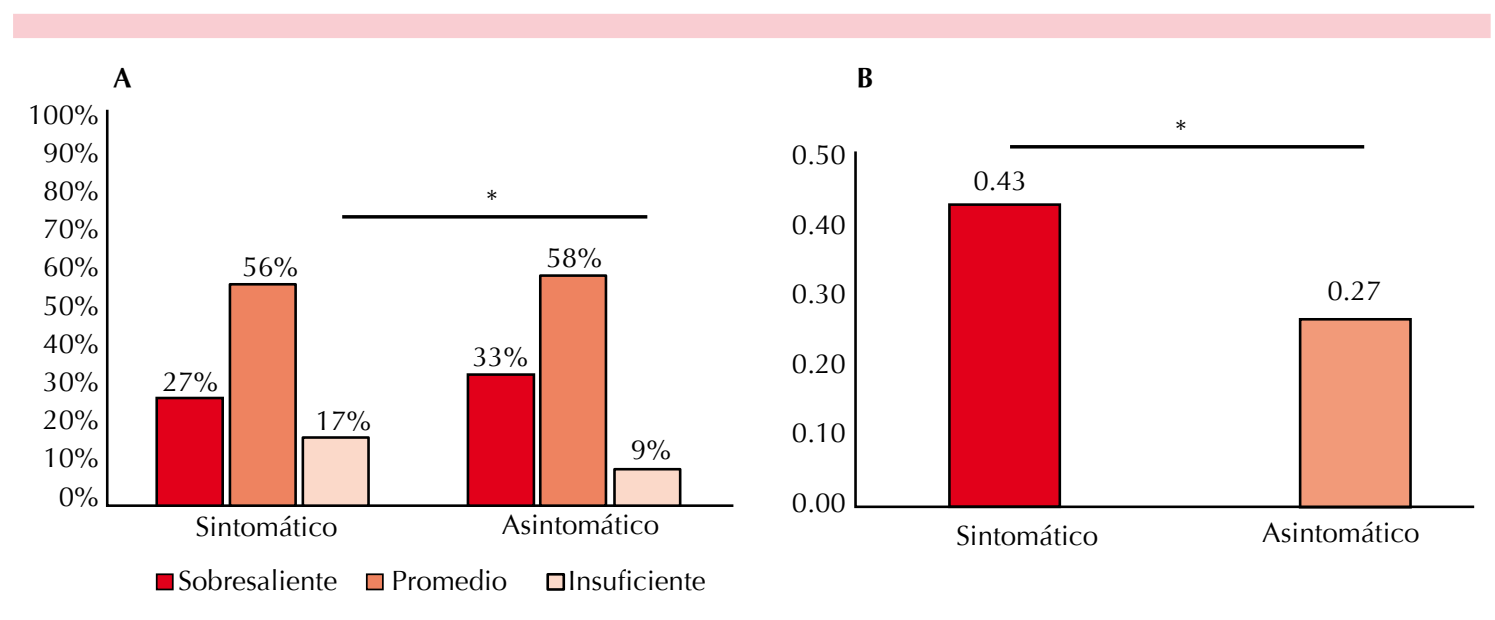

Figura 3. Rendimiento escolar y laboral. A. En los escolares el rendimiento se clasificó con una escala semicuantitativa. B. Entre los trabajadores, se calculó el número de amonestaciones acumuladas durante el periodo de estudio en la hoja de vida por persona en cada grupo.

${ }^{*} \mathrm{p}<0.05$.

$* * \mathrm{p}<0.01$. 
Cuadro 2. Efecto del tratamiento farmacológico

\begin{tabular}{lcccc}
\hline & Sintomático con tratamiento & Sintomático sin tratamiento & Asintomático & p \\
& 1.8 & 2.9 & 1.2 & 0.04 \\
Ausentismo escolar & $12 \%$ & $18 \%$ & $9 \%$ & 0.04 \\
Reprobado & 0.33 & 0.42 & 0.27 & 0.03 \\
Amonestaciones en los trabajadores & & &
\end{tabular}

La p representa la diferencia estadística entre el grupo sintomático con y sin tratamiento.

asma son una causa importante de ausentismo y bajo rendimiento escolar; de igual manera, su efecto en la población adulta lleva a un rendimiento laboral bajo. El tratamiento adecuado permite a estos pacientes tener un mejor control sintomático, lo que además mejora de manera importante el rendimiento laboral y académico de los pacientes, con resultados similares a los del grupo asintomático. La falta de asociación con ausentismo en la población adulta puede deberse a las costumbres sociales y culturales de nuestra población en la que a veces el temor a ser despedidos se antepone a la salud; sin embargo, el mayor número de amonestaciones refleja que en esta población el efecto socio-laboral de estas enfermedades también es importante.

La revisión de los artículos publicados permite concluir que la mayor parte de las evaluaciones económicas demuestra que la rinitis y asma tienen un costo económico alto por el tratamiento farmacológico, el seguimiento médico requerido, las urgencias, etc. Aunque en muchos países parte de este gasto es asumido por el tercer pagador (sistema de salud), todas las evaluaciones económicas coinciden en que el gasto es mayor para el paciente, porque es quien asume los costos indirectos de la enfermedad (ausentismo laboral o escolar del paciente y de su grupo social), que según algunos estudios representa la mayor parte de los gastos asociados con la rinitis y el asma. ${ }^{17}$

Los resultados de este estudio indican que el tratamiento farmacológico adecuado puede lle- var a un mejor control sintomático; no obstante, también observamos que aunque la mayoría de los pacientes tiene acceso a este tratamiento, la tasa de apego es muy baja, con lo que disminuye su efecto clínico, por lo que se necesitan campañas de promoción y prevención que permitan mayor concientización del problema al paciente.

Debido a que los artículos del tema se basan en estudios publicados en países de Europa y en Estados Unidos, se espera que la mayor parte analice evaluaciones económicas con sistemas de salud diferentes a las de los países latinoamericanos, por lo que las conclusiones de este tipo de estudios pueden variar de un lugar a otro según las características de cada población: por ejemplo, el costo de horas laborales, el tipo de sistema académico, etc. De manera similar a nuestros resultados, entre las pocas evaluaciones latinoamericanas realizadas acerca de este tema, Velástegui y colaboradores observaron en Chile que los pacientes escolares con asma severa tenían el doble de gasto derivado de salud y mayor ausentismo escolar que los sujetos no asmáticos. ${ }^{15}$ Es recomendable que en cada país se realice una valoración en términos de su situación social y económica; por esta razón nuestros resultados son importantes para Latinoamérica, porque exploran el efecto de la rinitis y el asma en una población con condiciones sociodemográficas similares a las reportadas en las principales ciudades tropicales de la región que tal vez se asemejen mejor a las realidades socioeconómicas, en comparación con los estudios provenientes de Estados Unidos o Europa. 
Nuestro estudio tiene varias limitaciones secundarias a su diseño observacional y la ausencia de cegamiento. Observamos que la población mayor de 18 años que trabajaba en las instituciones era mayoritariamente masculina; sin embargo, este sesgo no afecta de manera importante los desenlaces evaluados porque se contó con una muestra representativa de ambos sexos en los diferentes grupos. Entre las fortalezas está la medición sistemática de variables de evaluación objetivas, los cuestionarios los Ilenaron los pacientes con la asesoría de personal capacitado en el área de la salud (médicos, enfermeras o auxiliares de enfermería), lo que permitió un seguimiento adecuado y confiable de los parámetros evaluados. La selección de matemáticas y español como parámetros de evaluación del rendimiento escolar tiene algunas limitaciones; la variabilidad en los conocimientos de los maestros, las estrategias educativas usadas y las destrezas de los alumnos podían influir en los resultados de las notas. Sin embargo, estas variables se ven compensadas por el hecho de que todos los niños incluidos en cada plantel estaban sujetos a estas variables y la variable diferencial fue la existencia o no de síntomas respiratorios. Además, el mejor rendimiento entre todos los niños con asma, rinitis o ambas que recibían medicación en comparación con los que no tenían tratamiento refuerza la hipótesis de que la menor productividad encontrada entre los niños con enfermedades respiratorias se debía, en gran parte, a la enfermedad y no a otros factores, como el método evaluativo de cada institución.

En resumen, observamos que la rinitis y el asma son importantes factores de riesgo de ausentismo escolar y bajo desempeño laboral en población infantil y adulta; sin embargo, el efecto de estas enfermedades puede reducirse con un tratamiento adecuado. Es necesario realizar estudios de promoción y prevención para concientizar al paciente y a las instituciones de la importancia que tiene un buen control clínico de estas enfermedades.

\section{Agradecimientos}

Este artículo fue financiado por el ministerio de salud de Colombia, el Grupo de Alergología Clínica y Experimental de la Universidad de Antioquia, La Universidad de Cartagena y la Fundación Hospital San Carlos, Bogotá, Colombia.

Nuestro agradecimiento a las instituciones por su permiso y apoyo. A estudiantes, profesores y demás miembros de las instituciones que amablemente aceptaron participar en la realización del estudio.

\section{REFERENCIAS}

1. Dennis R, Caraballo L, Garcia E, Caballero A, et al. Asthma and other allergic conditions in Colombia: a study in 6 cities. Ann Allergy Asthma Immunol 2004;93:568-574.

2. Vergara C, Caraballo L. Asthma mortality in Columbia. Ann Allergy Asthma Immunol 1998;80:55-60.

3. Neffen H, Baena-Cagnani CE, Malka S, Sole D, et al. Asthma mortality in Latin America. J Investig Allergol Clin Immunol 1997;7:249-253.

4. Eder W, Ege MJ, von Mutius E. The asthma epidemic. N Engl J Med 2006;355:2226-2235.

5. Anandan C, Nurmatov U, van Schayck $O$, Sheikh A. Is the prevalence of asthma declining? Systematic review of epidemiological studies. Allergy 2010;65:152-167.

6. Fasce L, Tosca MA, Baroffio M, Olcese R, Ciprandi G. Atopy in wheezing infants always starts with monosensitization. Allergy Asthma Proc 2007;28:449-453.

7. Yepes-Núñez JJ, Gómez C, Espinoza Y, Cardona R. [The impact of subcutaneous immunotherapy with Dermatophagoides farinae and Dermatophagoides pteronyssinus on the quality of life of patients with allergic rhinitis and asthma]. Biomedica 2014;34:282-290.

8. Meltzer EO, Gross GN, Katial R, Storms WW. Allergic rhinitis substantially impacts patient quality of life: findings from the Nasal Allergy Survey Assessing Limitations. J Fam Pract 2012;61(2 Suppl):S5-10.

9. Jáuregui I, Mullol J, Dávila I, Ferrer M, et al. Allergic rhinitis and school performance. J Investig Allergol Clin Immunol 2009;19(Suppl 1):32-39.

10. Shedden A. Impact of nasal congestion on quality of life and work productivity in allergic rhinitis: findings from a large online survey. Treat Respir Med 2005;4:439-446. 
11. Caraballo L, Acevedo N. Allergy in the tropics: the impact of cross-reactivity between mites and ascaris. Front Biosci (Elite Ed) 2011;3:51-64.

12. Sanchez J, Diez S, Cardona R. Sensibilización a aeroalergenos en pacientes alérgicos de Medellín, Colombia. Revista Alergia México 2012;59:139-147.

13. Riechelmann $\mathrm{H}$, Europäischen Akademie für Allergie und Klinische Immunologie (EAACI) un der European Rhinologic Society (ERS). [Chronic Rhinosinusitis - EPOS 2012 Part I]. Laryngorhinootologie 2013;92:193-201; quiz 2-3.

14. Charlson ME, Pompei P, Ales KL, Mackenzie CR. A new method of classifying prognostic comorbidity in longitu- dinal studies: development and validation. J Chronic Dis 1987;40:373-383.

15. Velástegui $C$, Pérez-Canto $P$, Zárate $V$, Arenas $D$, et al. [Impact of asthma among primary attention children]. Rev Med Chil 2010;138:205-212.

16. Blanchette CM, Gutierrez B, Ory C, Chang E, Akazawa M. Economic burden in direct costs of concomitant chronic obstructive pulmonary disease and asthma in a Medicare Advantage population. J Manag Care Pharm 2008;14:176-185.

17. Baiardini I, Braido F, Tarantini F, Porcu A, et al. ARIAsuggested drugs for allergic rhinitis: what impact on quality of life? A GA2LEN review. Allergy 2008;63:660-669. 